\title{
Mehr Vertriebseffizienz durch CRM
}

Dass es Lücken im CRM-Einsatz (Customer Realationship Management) und in der Vertriebseffizienz gibt, belegt unter anderem eine neue Studie von Walter Zimmermann (Referent für Vertriebs- und Führungsmeetings, Redner für Kick-Offs und Veranstaltungen) zur Vertriebseffizienz in Unternehmen. Danach mangelt es nicht nur an der Prozessorientierung und Nutzung von CRM, sondern auch an Qualität und Anzahl der Vertriebsaktivitäten. Dies hat zur Folge, dass das Gros der Aktivitätenziele im Vertrieb nicht erreicht wird.

Vorhandene Vertriebsressourcen durch den Einsatz von CRM effizient zu nutzen, wird immer noch nicht durchgängig in den Unternehmen praktiziert. Dabei steigert strategisches CRM nachweislich den Vertriebserfolg.

Unternehmen schöpfen derzeit nur gut 65 \% der vorhandenen Vertriebsressourcen tatsächlich aus. Während in der
Produktion der Faktor Ressourceneinsatz weitgehend schon ausgereizt ist, gibt es gerade in Vertrieb und Dienstleistung noch viel Potenzial. Ein Grund für das Dilemma ist, dass im Kundenmanagement des Vertriebs häufig nicht die Kontakte mit dem richtigen Potenzial abgeschöpft werden und bei gleichem Zeitaufwand beispielsweise Kunden mit geringerem Potenzial bevorzugt werden. Auch Zeitpunkt und Abwicklung von Kundengesprächen in der Akquise, der Umgang mit der Daten-Mobilität im Vertrieb oder die richtige Datennutzung lassen zu wünschen übrig, weil CRM-Tools beispielsweise nicht richtig eingesetzt werden und wertvolle Kundenhistorien so im Datennirwana der Unternehmen schlummern.

SfP] ${ }^{*}$ www.springerprofessional.de/3690362

\section{Kleinstkapitalgesellschaften profitieren vom MicroBilG}

Bereits für Jahresabschlüsse zum 31.12.2012 können Kleinstkapitalgesellschaften die Regelungen des Kleinstkapitalgesellschaften-Bilanzrechtsänderungsgesetzes - kurz: MicroBilG in Anspruch nehmen. So können sie z. B. auf die Erstellung eines Anhangs zur Bilanz verzichten, wenn bestimmte Angaben (u. a. Haftungsverhältnisse) unter der Bilanz ausgewiesen werden. Weitere Vereinfachungen für diese Unternehmen gibt es im Bereich der Offenlegungspflichten und der Darstellungstiefe des Jahresabschlusses.

Zielsetzung des Gesetzes ist es, besonders kleine Kapitalgesellschaften von den derzeit umfangreichen Vorgaben für die Rechnungslegung auf EU-Ebene zu entlasten. Entlastun- gen für Einzelkaufleute waren bereits im Bilanzrechtsmodernisierungsgesetz enthalten. Nun gelten Vereinfachungen auch für Kleinstkapitalgesellschaften, die an zwei aufeinander folgenden Abschlussstichtagen zwei der drei nachfolgenden Merkmale nicht überschreiten:

- Nettoumsatzerlöse bis 700.000 Euro,

- Bilanzsumme bis 350.000 Euro sowie

- durchschnittlich 10 beschäftigte Mitarbeiter.

Die Neuregelungen finden bereits für alle Geschäftsjahre Anwendung, deren Abschlussstichtag nach dem 30.12.2012 liegt.

SfP $^{*}$ www.springerprofessional.de/3733938 